\title{
Life Satisfaction of Chinese Grandmothers: The Impact of Grandparenting Role Changes
}

\section{LOU VIVIAN W. Q.}

Department of Social Work \& Social Administration, Sau Po Centre on Ageing, The University of Hong Kong, Hong Kong

This article examines the impact of grandparenting role changes on life satisfaction among Chinese grandmothers in Hong Kong. Study participants included 166 grandmothers whose youngest grandchildren were older than twelve years. The grandmothers were recruited from elderly service users in different geographic areas in Hong Kong. Trained research assistants conducted face-to-face interviews using standardized scales. It was found that a reduced advisory role for grandmothers had a negative impact on their life satisfaction after controlling for their demographic characteristics, social support, and health status. Implications for policies on intergenerational programme and future research in a Chinese context are discussed.

KEYTERMS life satisfaction, grandmothers, grandparenting role change, Hong Kong, Chinese

This research project is supported by a Direct Grant from The Chinese University of Hong Kong. Ref. No. 2020824. 
Vivian W. Q. Lou, Ph.D., is Assistant Professor, Department of Social Work \& Social Administration, Associate Director, Sau Po Centre on Aging, The University of Hong Kong, Hong Kong; e-mail: wlou@hku.hk

Address correspondence to Dr. Vivian W. Q. Lou, Department of Social Work \& Social Administration, The University of Hong Kong, Pokfulam Road, Hong Kong. Email: wlou@hku.hk

\section{INTRODUCTION}

The life satisfaction of older adults has been proven to be a culturally embedded phenomenon. In a Chinese context, not only the conceptualization of life satisfaction but also the factors associated with life satisfaction were found to be more associated with relational and interpersonal aspects of life (Chou \& Chi, 1999; Kang et al., 2003; Lou et al., 2008). An aging population, including demographic changes from a prolonged life expectancy, has provided more opportunities for the elderly to play grandparenting roles. In traditional Chinese culture, a clear division of labor is developed through established and maintained kinship networks and relations. For example, males (fathers and sons) are expected to take care of things related to people outside of the family and also work hard to support the family, while females (mothers and daughters) take active roles in keeping the generations linked together. Accordingly, grandmothers are expected to play active roles in interacting with their grandchildren to serve as kin-keepers. A recent study 
among Hong Kong Chinese grandparents showed that grandmothers frequently

communicated with and made visits to their adolescent grandchildren (Lou, 2008).

Therefore, Chinese grandmothers actively take part in grandparenting roles.

Studies on grandmotherhood and life satisfaction have not reached consensus. On

one hand, taking up grandparenting roles has been found to be beneficial and positively related to the life satisfaction of the grandparents (Kreindler, 1994; Lou, 2010). On the other hand, grandmothers have been found to develop stress while taking up grandparenting roles and to be at risk of physical ill-health and psychological distress (Landry et al., 2005; Gerard et al., 2006). One hypothesis argued that the optimal level of grandmother involvement is taking up a moderate level of grandparenting but not serving as full-time caregivers (Cherlin \&Furstenberg, 1992; Schneiders, 1994). A study on grandparents in Hong Kong found that involvement in two to six years of taking care of grandchildren served as the optimal involvement of grandparents, which was positively associated with the life satisfaction of grandparents (Liang, 2006).

However, what is a "moderate level”? Grandparent involvement should not be a static concept but rather a dynamic process affected by contextual factors (Strom \& Strom, 1997). One important contextual factor is the developmental stages of grandchildren. Grandparenting roles will change according to the ages of the 
grandchildren (Crosnoe \& Elder, 2002; Silverstein \& Marenco, 2001). Literature on the

moderate level of grandparenting largely focuses on grandchildren in their childhood stage, while a focus on grandchildren at the adolescent and older stages is neglected (Cherlin \& Furstenberg, 1992; Liang, 2006). Another important contextual factor is social and economic changes on grandparenting role expectations in a particular cultural context. In Hong Kong, when extended family is not as desirable as before, older women might find a lack of opportunity to play grandparenting roles in providing hands-on support (Mjelde-Mossey, 2007). In addition, values and attitudes toward the traditional family hierarchy and respect for older people also have been challenged (Chow \& Lum, 2008).

It would be worthwhile to examine the impact of grandparenting role changes on the life satisfaction of grandmothers with adolescent or adult grandchildren to expand the scope of research on the moderate level of grandparenting with a contextual factor-driven scope.

\section{LITERATURE REVIEW}

\section{Theoretical Approaches to Grandparenting Role Changes}

According to the kin-keeper hypothesis regarding gender and grandparents, grandmothers traditionally are expected to serve as the kin-keepers of the family. The 
hypothesis further indicates that, by holding families together, women are the glue of a family. In this regard, females are expected to have closer relations with all family members, including grandchildren (Bubas, 2001). Such an understanding seems to fit the context of Chinese culture in which mothers are expected to take care of internal family issues, while the males in a family are expected to take responsibility for external concerns (e.g., financial resources, relationships with networks beyond core family members, etc.). A recent study in Hong Kong supported the kin-keeper perspective by finding that grandmothers were more active than grandfathers in communicating and maintaining relationships with grandchildren (Lou, 2008). On top of that, the symbolic interaction theory explains why initial interpersonal interactions are sustained: people like to maintain positive self-meaning generated from interpersonal inactions. Therefore if initial interaction brings positive self-identity, including self-meaning and selffulfillment, people tend to continue to invest in such relationships (Stryker \& Burke, 2000).

Since both grandchildren and grandmothers are developing along their own life-span developmental stages, grandparenting roles are expected to change to accommodate changes experienced by both parties and in response to external changes such as family environment and societal expectations. The social role perspective helps to explain such a 
dynamic relationship (Cottrell Jr., 1942). According to social role theory, the grandparenting role reflects social and cultural norms and expectations toward grandparents in a particular context. It also suggests that the grandparenting role is expected to adapt to social and cultural changes in a particular context. If a person is able to change his or her role in regard to family, gender, and the developmental stages of grandchildren according to environmental demands, he or she would obtain a positive identity and self-concept, which will lead to psychological well-being. Otherwise, psychological distress is expected (Cottrell Jr., 1942; Phillips, 1957).

It is obvious that when grandchildren grow into adolescence and young adulthood, their needs is different than when they were children (Crosnoe \& Elder, 2002). In addition, the modernization process has had a great impact on grandmothers in China as compared to grandfathers in regard to the expectations placed on grandparenthood (Mjelde-Mossey, 2007).

\section{Chinese Grandmothers: Tensions of Changing Grandparenting Roles}

For current Hong Kong Chinese grandmothers, the tension of changing grandparenting roles is generated from the internalized role expectations of a traditional "good grandmother" and the expectations for grandmothers in a changing social cultural context. In Chinese tradition, social expectations toward old age are clearly defined as 
compared to Western societies (Mjelde-Mossey, 2007). The current cohort of Hong Kong grandmothers have been exposed to a high-context cultural tradition that emphasizes family, hierarchy, and intergeneration relations (Yang, 1995; Chow, 1996).

Traditionally, the hierarchical relationship in the family puts women in a delicate position. The inferior position of women was constructed within ancient Chinese culture, which expected Chinese women to obey the men in her family (i.e., obey her father before getting married, obey her husband after marriage, and obey her son after being widowed). On the other side of the coin, women act as the glue of the family, serving as kin-keepers and enjoying implicit power within a family (Croll, 1995). Also, grandmothers are expected to be actively involved in and contribute to familial wellbeing through various means such as socializing grandchildren, helping with household chores, and providing financial help (e.g., Brian \& Logan, 2001; Li, 2001; Wong, 2004; Wu \& Sun, 2003). In addition, activities both directly with grandchildren and indirectly through the parent generation are considered significant. In return, grandmothers enjoy a legitimate high status in a family, symbolizing family authority and standing at the centre of family relations (Brian \& Logan, 2001; Chan et al., 2000; Strom et al., 1999). Even though certain developmental stages of grandchildren reduce the need for hands-on help, daily care and instrumental help by grandparents are not excluded from normative 
expectations. Grandparenting roles are associated with caring and love within intergenerational relations, and the life meaning and identity of grandmothers in Chinese culture.

In Hong Kong, the rapid social changes of the past decades have caused potential threats to traditional grandparenting role expectations. For example, families have fewer children than before. The family structure has changed and families have become smaller, with the average household size decreasing to three. More older adults either live alone or separate from their adult children and grandchildren. Therefore, grandparents have fewer opportunities to practice grandparenting roles. In fact, hiring a domestic helper to take care of children is considered a valid choice among younger generations. In addition, the social attitude toward filial piety has been redefined under the changing ecology—family ideologies such as the hierarchical status of grandparents are being challenged (Census and Statistics Department, 2003; Census and Statistics Department, 2008; Chow, 2001; Kwan et al., 2003; Tam, 2001). A recent study among Hong Kong grandparents showed that grandparents continue to play roles as advisors, educators, daily life helpers, and "fire fighters" (intervene when their children have family crisis such as financial difficulties, emergencies, and family violence) in grandparenting relations. They have 
also learned new roles in response to familial changes, such as supervising domestic helpers taking care of grandchildren (Tam, 2001).

This seems to suggest that demographic changes and the modernization process have challenged normative grandmother roles in a particular culture context in Hong Kong. It also suggests that grandmothers are expected to be less involved in direct grandparenting roles, an idea that conflicts with traditional norms. However, it is unclear how such experiences would affect the psychological well-being of grandmothers. The present study aims to fill the gap by examining the impact of grandparenting role changes on grandmothers' life satisfaction.

\section{Chinese Women and Life Satisfaction}

Factors embedded in both the personal and interpersonal levels of people's lives are associated with life satisfaction. At the personal level, socio-economic characteristics are related to life satisfaction (Fernandez-Ballesteros et al., 2001). In addition, health and wealth are consistently found to be associated with life satisfaction. Ill-health and its associated consequences such as medical consultation, a sense of loss of control, and a sense of weakness impede people from feeling satisfied about their lives (Stolar et al., 1992). Financial constraints also influence life opportunities and are positively related to lower life satisfaction (Lipovčan et al., 2007). Studies of life satisfaction among Chinese 
people also argue that interpersonal variables play a significant role in life satisfaction

(Lou et al., 2008).

In regard to gender and life satisfaction, there is no consensus on the pathways to

life satisfaction. Liang's model in the 1980s argued that men and women shared the same pathways to life satisfaction, and emphasized variables including education level, financial satisfaction, health, objective integration, and subjective integration (Liang, 1982). However, in 2005, Bourque argued that even though men and women shared similar pathways to life satisfaction, social support was more important to women, which led to perceived control (Bourque et al., 2005). A longitudinal study of Hong Kong elders found that social support was a predictive factor in life satisfaction for men and women (Chou \& Chi, 1999). In this study, variables such as demographic characteristics, social support, tradition, self-rated health, and self-rated financial status were considered in multi-variate analyses.

\section{METHODS}

\section{Sample}

The sample for the current study was recruited from social service organizations that provide services for the elderly in Hong Kong. Due to a lack of official statistics on grandparents in Hong Kong, a multistage sampling method was adopted to recruit 
respondents from diverse backgrounds. In stage one, strata sampling was used to select 90 service units from a list of elderly residential homes and senior centers in Hong Kong. Two strata were used: the nature of services (residential or community-based service) and geographic area (Hong Kong Island, Kowloon, and New Territory). Fifteen service units were randomly selected in each of the six categories (e.g., a community center in Hong Kong Island, a residential home in New Territory, etc.). Invitation letters and a brief introduction of the study were sent to the administrators of each selected service unit. From the 90 letters sent, 17 declined to participate and 21 were invalidated due to renovation, temporary suspension of visiting due to a flu epidemic, and invalid address due to relocation. The success rate was 75 percent.

In stage two, quota sampling was adopted to recruit respondents. Within each participating residential home or senior center, six respondents were set as the maximum number of participants to be interviewed to ensure that the study sample came from various geographic areas. A trained interviewer visited each center or residential home that agreed to participate in the study. At the centers or residential homes, trained interviewers approached elderly people and asked whether they had a grandchild aged 12 or older. Those who met the criteria were invited, and those who gave consent were interviewed using the standardized questionnaire. 


\section{Measures}

Life Satisfaction. The Life Satisfaction Scale-Chinese, an eight-item scale measuring domain-specific life satisfaction for Chinese elders, was adopted (Lou et al., 2008). The scale score was computed by summing the item responses $(1=$ Yes, $0=$ No/Not Applicable), with a theoretical range from 0 to 8 . A higher score is associated with a higher level of life satisfaction. The reliability of the scale in the present study was satisfactory, as indicated by a Chronbach’s alpha of .89.

Grandparenting Role Change. The grandparenting role was measured by a 24-item grandparenting role list developed from the literature. Literature on grandparenting roles found that these roles can be conceptualized along dimensions that reflect an underlying process or meaning. In the early 1960s, a classic study by Neugarten and Weinstein (1964) found that grandparents played various roles such as fun seeking, formal care, reservoirs of family wisdom, and surrogate parents. Recent studies also found similar grandparenting role dimensions such as daily life helpers, advisors, educators, and tradition transmitters (e.g., Harwood, 2001; Wiscott \& Kopera-Frye, 2000). Since the present study targeted grandparents with adolescent and adult grandchildren, the surrogate parent role was excluded. Therefore, the 24-item list included grandparenting role dimensions such as daily life helpers, advisors, educators, and helpers during crisis 
based on the ideas above. One more cultural and contextual specific role dimension supervisors' role in providing supervision to domestic helpers taking care of grandchildren - was added (Lou, 2005; Tam, 2001).

Each respondent was asked to indicate his or her behavioral frequency (never, seldom, sometimes, or often) on the 24-item grandparenting role list at two independent reference times. One reference time specified the time when their youngest grandchild was aged older than twelve years old (now). The second reference time specified ten years ago, when their grandchildren were in childhood. Exploratory factor analysis, based on responses at the "now" reference time, was computed by using the principle component method with varimax rotation. Five grandparenting roles, including advisors (five items), educators (eight items), daily life helpers (five items), "fire fighters” (three items) and supervisors (three items), explained 67 percent of the total variance. Exploratory factor analysis, based on the ten years ago when their grandchildren were in their childhood reference time, was also computed and generated similar factorial results that supported the stability of the five roles structure.

In the present study, the reliabilities of the five sub-scales, among responses at the now reference time and at the ten years ago reference time, were satisfactory as indicated by Cronbach’s alpha ranging from .60 to .92 (Table 1). Therefore, sub-scale scores were 
computed by dividing the sum of item scores with number of items in a corresponding sub-scale, which lead to a theoretical range of 1 to 4 . Higher subscale score indicates higher attribute for each subscale.

[Insert Table 1 about here]

Under each grandparenting role dimension, participants were categorized into two groups: respondents that reported no grandparenting role change (NCG) as compared to ten years ago (when their grandchildren were in their childhood) and respondents that reported a grandparenting role change (CG) as compared to ten years ago (when their grandchildren were in their childhood). The NCG/CG group was computed by calculating differences between sub-scale scores of the two time references for each of the five role dimensions independently. For those respondents who reported a decreased score in playing a specific grandparenting role when comparing the two time references, they grouped as CG. For those respondents who reported an unchanged score in playing a specific grandparenting role when comparing the two time references, they were grouped as NCG. Since there were only two to four respondents who reported an increase in behavioral frequency in playing roles such as educator, advisor, and supervisor, they were not included in the data analyses for the present study. 
Social Support Network. The participants’ social support network was measured using the Lubben Social Network Scale (LSNS) (Chi \& Boey, 1993; Lubben, 1988). This is a ten-item scale that measures the social support from family, friends, and interdependent relationships of the older respondents. The raw scores ranged from 0 to 50, with a higher score relating to a higher level of social support. The reliability of the total score was satisfactory, as indicated by Cronbach's alpha equal to 0.78 .

Chinese Family Tradition. Chinese family tradition was measured by the Family Tradition Scale for the Elderly (FTSE) (Mjelde-Mossey et al., 2005). FTSE contains nine items that measure the family tradition tendencies of Chinese elders. The total score is a sum of the item scores, with a theoretical range of 0 to 9 . A higher score is associated with a greater inclination toward family tradition.

Self-rated Health. Self-rated health was assessed by a single item question, "How would you rate your overall health at the present time?” Results were on a five-point scale (poor, fair, good, very good, and excellent). Self-rated health is a popular area of study among variables that assess health conditions for older adults, including the Chinese population (Bjørner et al., 1996; Chi \& Boey, 1993).

Financial Status. Self-rated financial adequacy was measured by a single item question, "Do you think that you have enough money to cover your daily expenses?" 
Results were on a three-point scale (adequate, just enough, and inadequate). This scale is used widely to measure the financial status of the elderly and has satisfactory reliability and validity among elderly Chinese people (Chou \& Chi, 2002).

Demographic Characteristics. The study gathered demographic data such as age, gender, marital status, level of education, living arrangement, and religious affiliation.

\section{Procedures}

Trained interviewers conducted face-to-face interviews to collect data.

Interviewers were recruited from among undergraduate students at one university in Hong Kong. Each interview was conducted in a private room or corner of a community center or residential home to ensure confidentiality. Each interview lasted about 30 minutes. The research ethics committee of the Chinese University of Hong Kong approved the study.

\section{Data Analysis}

Descriptive analyses were computed to show sample characteristics. Then differences between the perceived role changes in life satisfaction were computed. Last, a multiple regression analysis was conducted on the life satisfaction of grandmothers.

\section{FINDINGS}

\section{Respondents' Characteristics}


Among the 166 respondents, ages ranged from 64 to 101, with a mean age of 79.2 years. Approximately 60 percent had not received any formal education, and approximately 40 percent were married during the time of the interview. Nearly half the respondents lived in residential homes, and approximately 12 percent were living alone in the community. Half said they held religious beliefs such as Christian, Catholic, Daoist, Buddhist, etc. The sample characteristics are listed in Table 2.

[insert Table 2 about here]

\section{Grandparenting Role Changes}

Table 3 illustrates the differences found in grandparenting roles between the two reference times. The results show that, in the experience of grandmothers, the frequency of roles played decreased as compared to when their grandchildren were in their childhood. When their grandchildren were in their childhood, the most frequent role the grandmothers played was daily life helper followed by educator and advisor. When their grandchildren became adolescents or young adults, the sequence of frequently played roles remained the same even though the frequency of playing the roles lessened.

[insert Table 3 about here]

\section{Grandparenting Role Changes and Life Satisfaction}


Table 4 shows the effects on life satisfaction for two groups of respondents: those that reported no grandparenting role change (NCG) and those that reported a decrease in the grandparenting role as compared to ten years ago (CG). It was found that reduced behavioral frequency as advisor was associated to a decreased level of life satisfaction among grandmothers. On the contrary, reduced behavioral frequencies as educators and “fire fighters” were associated to an increased level of life satisfaction among grandmothers. Reduced behavioral frequencies as daily life helpers and supervisors were not significantly associated with the level of life satisfaction of grandmothers.

[insert Table 4 about here]

\section{Life Satisfaction of Grandmothers - The Impact of Grandparenting Role Changes}

Hierarchical multiple regressions were computed to examine the impact of grandparenting role changes on the life satisfaction of grandmothers (Table 5). Demographic variables and variables found to make significant contributions to life satisfaction for Chinese older adults (including age, marital status, living alone or not, living in residential homes or not, social support network, self-rated health, inclination toward Chinese family tradition, and self-rated financial adequacy) were entered in Models One and Two. Then, grandparenting role changes were entered in Model Three.

The results from Model Three showed that reduced behavioral frequency in the advisory 
role was significantly associated with less life satisfaction, which explained an extra 5

percent of the total variance $\left(\mathrm{R}^{2}\right.$ change $\left.=.05, p<.05\right)$ on the life satisfaction of

grandmothers. In general, grandmothers who lived in the community, were inclined

toward Chinese family traditions, had better self-rated health and financial opinions, and

had no decrease in behavioral frequency in playing an advisory role in grandparent-

grandchildren relationships were more likely to enjoy a higher level of life satisfaction.

[insert Table 5 about here]

\section{DISCUSSION}

The findings of the present study expand the knowledge of the moderate involvement of grandparents in the context of grandmother-adolescent/adult grandchildren in a Chinese context under social and cultural changes. In this study, moderate involvement and its relationship with life satisfaction depended on the nature of various roles in grandmother and adolescent/adult grandchildren relations.

\section{Grandparenting Role Change and Life Satisfaction among Chinese Grandmothers}

For those roles associated with direct help such as educator and daily life helper, a

reduced behavioral frequency was associated with a higher level of life satisfaction. One

possible explanation is that when grandchildren grow to adolescence, they become more independent and more mature in regard to self-regulated behaviors. Therefore, it is 
natural for grandparents to reduce their involvement in providing hands-on help, which is in line with social role change effects on expectations toward grandchildren and grandparents (Cottrell Jr., 1942; Crosnoe \& Elder, 2002). Grandmothers who experienced no change in behavioral frequencies were either overly committed to their roles or have had to take up heavy caregiving roles for grandchildren with special needs. Studies of Hong Kong’s adolescents showed that this generation perceived their parental behavior as being in control, which implies that some of the "good intended help and/or education" provided by older generations is not received well by adolescents (Shek, 2006).

For the advisory role, reduced behavioral frequency was found to be negatively related to life satisfaction. In other words, those grandmothers who reduced their advisory role in grandparenting were more likely to feel less satisfied with life. This finding can be explained by the tension between changed societal expectations and the traditional role expectations of the current cohort of Chinese grandmothers. When grandmothers reduce their role as advisors they might do it by force instead of by choice. For example, living apart from children and grandchildren reduces opportunities for older people to provide advice. Children do not seek advice actively from parents in regard to issues concerning the grandchildren (Kwan et al., 2003). Children and grandchildren do not see advice from 
grandparents as valuable and constructive, but may believe that older grandparents fail to understand the life circumstances of the young people (Chow \& Lum, 2008).

For "fire fighter" and supervisor roles, a lower behavioral frequency had no significant association with the life satisfaction of grandmothers. A general trend toward decreasing behavioral frequencies of these roles was observed, which was consistent with observations of the other three roles. One explanation is that these two roles are timelimited. Grandmothers only serve as "fire fighters" when there is a family crisis and only provide supervision of domestic helpers when needed.

In regard to the findings based on regression, it was observed in the present study that decreased behavioral frequency of the advisory role significantly lowered the life satisfaction of grandmothers. This observation can be explained by social role theory and symbolic interactions on identity formation (Cottrell Jr., 1942; Stryker \& Burke, 2000). It suggests that Chinese older women in Hong Kong are able to adjust their role expectations for serving as educators and daily life helpers according to the life-span developmental needs of grandchildren and changed social expectations. For those who fail to adjust, lower life satisfaction results. However, serving as advisors on grandchildren issues is an internalized identity for Chinese older women. During the process of providing advice, grandmothers build up positive life-meaning that they want 
to maintain and develop. When this meaning is threatened, life satisfaction is negatively

affected.

\section{Other Factors Affecting Life Satisfaction of Chinese Grandmothers}

In regard to other factors identified as contributing to the life satisfaction of grandmothers, the study revealed that living in a residential home is a hindering factor while inclination toward traditional family values and having better self-rated health and financial status were facilitating factors of life satisfaction, which is consistent with the literature (Chou \& Chi, 1999; Lou et al., 2008). The present study found that social support networks were not a significant factor. Further exploration revealed that the social support network variable was entangled with the living in residential home variable. Respondents who lived in residential homes showed a significantly lower level of social support compared to those who lived in the community (Mean difference 11.02, $t=8.35, p<.000)$. When the variable of living in a residential home was excluded from the regression model, social support became a significant contributing factor for life satisfaction.

\section{Contextualized Implications}

The results of the present study could have three implications for policy on intergenerational programs and future research in a Chinese context under social and 
economic change. First, social norms and expectations toward grandmothers are changing in the context of modernization. Chinese female identity is expected to be re-built so that a better quality of life can be achieved. Policy initiatives on gender role re-building at the societal, community, family, and individual levels are recommended. After ten years of a gender mainstreaming policy in Hong Kong, women still have a very disadvantaged status in Hong Kong, which is worth further policy and public attention (Tang \& Lou, 2009). Second, programs involving intergenerational exchanges and interactions within and outside the family boundary are recommended. The Elderly Commission initiated intergenerational cohesion programs years ago; it is recommended that these programs be continued and become more focused—for example, targeting women not living with children and grandchildren (Elderly Commission, 2001). In response, it is important for each generation to discuss and reflect on their expectations toward Chinese family traditions and intergenerational bonds. It is obvious that the Chinese community cannot afford the cost of totally losing traditions, but how to re-vision Chinese family tradition in the modernized context is a challenge for policy makers as well as the public (Chow \& Lum, 2008). Last but not least, while various grandparenting roles were found to have different impacts on life satisfaction, the findings were generated by one study only. 
Further studies using larger samples and a longitudinal design are suggested so grandparenting role changes can be observed more vigorously.

\section{Limitations}

The present study has several limitations. First, the study sample was nonrepresentative regardless of the fact that a multi-stage sampling procedure was adopted to enhance the diversity of the sample. In addition, although trained interviewers randomly approached older people at selected centers, selection bias would not be adequately controlled by using this approach. Moreover, elderly people with communication difficulties were excluded. Any attempt at generalizing the findings should be done with caution. Second, grandparenting role changes were measured by self-reported measures, with one measure based on retrospective evaluation. Reliability of one role subscale, supervisors role in particular, was low in this sample. Longitudinal studies on larger representative samples are recommended so that grandparenting role changes can be followed. 


\section{REFERENCES}

Bjørner, J. B., Kristensen, T. S., Orth-Gomér, K., \& Sullivan, M. (1996). Self-rated health: A useful concept in research, prevention and clinical medicine. Swedish Council for Planning and Coordination of Research, Stockholm (1966), p. 9, report no. 96.

Bourque, P., Pushkar, D., Bonneville, L., \& Beland, F. (2005). Contextual effects on life satisfaction of older men and women. Canadian Journal on Aging, Canada, University of Toronto Press. 24: 31-44.

Brian, J. S., \& Logan, J. (2001). Intergenerational relations: A comparison between Chinese and American families (in Chinese). Journal of Sociology. 2: 85-95.

Bubas, J. S. (2001). How gender moderates the grandparent-grandchild relationship: A comparison of kin-keeper and kin-selector theories. Journal of Family Issues, 22: 478-492.

Census and Statistics Department (2003). Thematic household survey report no. 14, Sharing of housework. Hong Kong: Census and Statistics Department, the HK SAR government.

Census and Statistics Department (2008). 2006 population by-census thematic report: Older persons. Hong Kong: Census and Statistics Department, the HK SAR government.

Chan, C. M. A., Phllips, D. R., Chi, I., Ho, S., Cheng, S. T. \& Cheung, J. (2000). The study of the quality of life of elderly residents in the community in Hong Kong. Report submitted to the Health and Welfare Bureau, Hong Kong government. 
Cherlin, A., \& Furstenberg, F. (1992). The new American grandparent: A place in the family, a life apart. Harvard University Press.

Chi, I., \& Boey, K. W. (1993). Hong Kong validation of measuring instruments of mental health status of the elderly. Clinical Gerontologist, 13(4): 35-51.

Chou, K. L., \& Chi, I. (1999). Determinants of life satisfaction in Hong Kong Chinese elderly: A longitudinal study. Aging and Mental Health, 3(4): 328-335.

Chou, K. L., \& Chi, I. (2002). Financial strain and life satisfaction in Hong Kong elderly Chinese: Moderating effect of life management strategies including selection, optimization, and compensation. Aging and Mental Health, 6(2): 172-177.

Chow, N. W. S. (1996). The Chinese society and family policy for Hong Kong. Marriage and Family Review, 22: 55-72.

Chow, N. W. S. (2001). The practice of filial piety among the Chinese in Hong Kong. In I. Chi, N. Chappell and J. Lubben, Elderly Chinese in pacific Rim countries (pp. 125-136). Hong Kong: The University of Hong Kong Press.

Chow, N. W., \& Lum, T. (2008). Trends in family attitudes and values in Hong Kong. Hong Kong: Department of Social Work \& Social Administration, The University of Hong Kong.

Cottrell, L. Jr. (1942). The adjustment of the individual to his age and sex roles. American Sociological Review, 7: 617-620.

Croll, E. (1995). Changing identities of Chinese women: Rhetoric, experience, and selfperception in twentieth-century China. London: Zed Books Ltd. 
Crosnoe, R., \& Elder, G. H. Jr. (2002). Life course transitions, the generational stake, and grandparent-grandchild relationships. Journal of Marriage and Family. 64: 10891096.

Elderly Commission (2001). Report on healthy aging. Hong Kong: Elderly Commission. Fernandez-Ballesteros, R., Dolores, Z. M., \& Angel, R. M. (2001). The contribution of socio-demographic and psychosocial factors to life satisfaction. Aging and Society. 21: $25-43$.

Gerard, J. M., Landry-Meyer, L., \& Roe, J. G. (2006). Grandparents raising grandchildren: The role of social support in coping with caregiving challenges. International Journal of Aging and Human Development. 62: 359-383.

Harwood, J. (2001). Comparing grandchildren and grandparents' stake in their relationship. International Journal of Aging and Human Development, 53, 195-210.

Kang, S. M., Shaver, P. R., Sue, S., Min, K, H., Jing, H. (2003). Culture-specific patterns in the prediction of life satisfaction: Roles of emotion, relationship quality, and selfesteem. Personality and Social Psychology Bulletin. 29: 1596-1608.

Kreindler, L. K. (1994). Grandparents: The relationship between life satisfaction and the grandparenting role. Unpublished Ph.D. Thesis. New York: Pace University.

Kwan, Y., Cheung, C., \& Ng. S. H. (2003). Revisit of the filial piety concept among the young, the adult, and the old in Beijing, Guangzhou, Hong Kong, Nanjing, Shanghai, Xiamen, and Xian. Dept. of Applied Social Studies, City University of Hong Kong, Hong Kong.

Landry Meyer, L., Gerard, J. M., Guzell, J. R., \& Caputo, R. K. (2005). Caregiver stress among grandparents raising grandchildren: The functional role of social support. 
Marriage and Family Review. 37: 171-190.

Li, Q. X. (2001). Grandparents parenting in pre-school education. Journal of Pre-school Education. 3: 16-17. (in Chinese)

Liang, J. (1982). Sex differences in life satisfaction among the elderly. Journal of Gerontology. 37(1): 100.

Liang, Y. (2006). Impacts of grandparenting on life satisfaction of the Chinese elderly in Hong Kong, Unpublished MSoSc Thesis. Hong Kong: University of Hong Kong.

Lipovčan, L., Brkljacić, T., Sakić, V. (2007). Monthly income and subjective well-being of Croatian citizens. Croatian Medical Journal. 48: 727-733.

Lou, V. W. Q. (2005). Grandparenting roles played by older people in Hong Kong. $13^{\text {th }}$ Annual Congress of Gerontology, Hong Kong Association of Gerontology. Hong Kong, Hong Kong Association of Gerontology.

Lou, V. W. Q. (2008). Gender and grandparent-grandchild relations in Hong Kong. Gender \& Family in East Asia International Conference 2008. Hong Kong: Gender Study Research Center, The Chinese University of Hong Kong.

Lou, V. W. Q. (2010). Life satisfaction of older adults in Hong Kong: The role of social support from grandchildren. Social Indicators Research, 95(3): 377-391.

Lou, V. W. Q., Chi, I., Mjelde-Mossey, L. A. (2008). Development and validation of a life satisfaction scale for Chinese elders. International journal of aging and human development. 67: 149-70.

Lubben, J. (1988). Assessing social networks among elderly populations. Family \& Community Health. 11: 42. 
Mjelde-Mossey, L. (2007). Cultural and demographic changes and their effects upon the traditional grandparent role for Chinese elders. Journal of Human Behavior in the Social Environment. 16: 107-120.

Mjelde-Mossey, L. A., Chi, I., Lou, V. W. Q. (2005). Assessing tradition in Chinese elders living in a changing social environment: Implications for social work practice. Journal of Human Behavior in the Social Environment. 11: 41-57.

Neugarten, B. L. \& Weinstein, K. K. (1964). The changing American grandparent. Journal of Marriage and the Family, 26: 199-204.

Phillips, B. (1957). A role theory approach to adjustment in old age. American Sociological Review. 22: 212-217.

Schneiders, B. F. B. (1994). Impact on grandparents of providing various levels of caregiving for grandchildren. Unpublished Ph.D. Thesis. Virginia: Virginia Commonwealth University.

Shek, D. (2006). Perceived parental behavioral control and psychological control in Chinese adolescents in Hong Kong. The American Journal of Family Therapy. 34: 163-176.

Silverstein, M., \& Marenco, A. (2001). How Americans enact the grandparent role across the family life course. Journal of Family Issues. 22: 493.

Stolar, G. E., MacEntee, M. I., Hill, P. (1992). Seniors’ assessment of their health and life satisfaction: The case for contextual evaluation. International journal of aging \& human development. 35: 305-317.

Strom, R., \& Strom, S. (1997). Building a theory of grandparent development. International Journal of Aging \& Human Development. 45: 255-286. 
Strom, R., Strom, S., Wang, C., Shen, Y., Griswold, D., Chan, H., Yang, C. (1999). Grandparents in the United States and the Republic of China: A comparison of generations and cultures. Aging and Human Development. 49(4): 279-317.

Stryker, S., \& Burke, P. J. (2000). The past, present, and future of an identity theory. Social Psychology Quarterly. 63: 284-297.

Tam, V. C. W. (2001). A family ecological analysis of child care use in Hong Kong. Children \& Society. 15: 181-192.

Tang, K. L. \& Lou, V. W. Q. (2009). Social welfare and women: The dominant approach and its critique. In F. M. Cheung, \& E. Holroyd (Eds.), Mainstreaming gender in Hong Kong society (pp. 181-210). Hong Kong: The Chinese University Press.

Wiscott, R. \& Kopera-Frye, K. (2000). Sharing of culture: Adult grandchildren’s perceptions of intergenerational relations. International Journal of Aging and Human Development, 51, 199-215.

Wong, S. X. (2004). Changes of population and birth policy and its impact on intergenerational relations. Population \& Economics. 4: 9-14. (in Chinese)

Wu, L. L. \& Sun, Y. P. (2003). Family intergenerational exchange and its impacts on mental health of the elder adults. Journal of Chinese Gerontology. 23: 803-804. (in Chinese).

Yang, G. S. (1995). Social orientation of Chinese people. In T. Y. Lin, W. S. Tseng, \& E. Yeh, K. (Eds.), Chinese Societies and Mental Health (pp. 19-39). Hong Kong: Oxford University Press. 
TABLE 1. Reliabilities of Grandparenting Role Subscales (N=166)

\begin{tabular}{|c|c|c|c|}
\hline & $\begin{array}{l}\text { Number of } \\
\text { items }\end{array}$ & $\begin{array}{c}\text { Ten years ago when } \\
\text { their grandchildren } \\
\text { were in their childhood }\end{array}$ & $\begin{array}{c}\text { When grandchildren } \\
\text { were grown to } \\
\text { adolescence or young } \\
\text { adulthood (now) }\end{array}$ \\
\hline Advisors & 5 & .87 & .87 \\
\hline Educators & 8 & .92 & .90 \\
\hline $\begin{array}{l}\text { Daily life } \\
\text { helpers }\end{array}$ & 5 & .84 & .83 \\
\hline "Fire fighters" & 3 & .73 & .68 \\
\hline Supervisors & 3 & .60 & .63 \\
\hline
\end{tabular}


TABLE 2. Sample Characteristics of Respondents

\begin{tabular}{lll}
\hline & Frequency & Percentage (\%) \\
\hline Female & 166 & 100 \\
Age (Mean = 79.2, SD =6.9) & 12 & 7.2 \\
$60-69$ & 77 & 46.4 \\
$70-79$ & 77 & 46.4 \\
80 or above & & \\
Level of education & 103 & 62.0 \\
No formal education & 63 & 38.0 \\
Formal education & & 39.2 \\
Marital status & 65 & 60.8 \\
Currently married & 101 & 12.0 \\
Currently not married & & 40.4 \\
Living arrangement & 20 & 47.6 \\
Living alone in the community & 67 & 43.4 \\
Living in the community with others & 79 & 56.6 \\
Living in residential homes & & \\
Religious affiliation & 72 & \\
No & 94 & \\
Yes & &
\end{tabular}


TABLE 3. Grandparenting Role Changes - Comparing When Grandchildren Were Grown to Adolescence or Young Adulthood (Now) and Ten Years Ago When Their Grandchildren Were in Their Childhood (N=166)

\begin{tabular}{lcccc}
\hline & \multicolumn{2}{c}{$\begin{array}{c}\text { Ten years ago when their } \\
\text { grandchildren were in their } \\
\text { childhood }\end{array}$} & $\begin{array}{c}\text { When grandchildren were grown } \\
\text { to adolescence or young } \\
\text { adulthood (now) }\end{array}$ \\
\cline { 2 - 5 } & Mean & SD & Mean & SD \\
\hline Daily life helpers & 2.34 & 0.89 & 1.70 & 0.48 \\
Educators & 1.97 & 0.80 & 1.68 & 0.60 \\
Advisors & 1.80 & 0.76 & 1.58 & 0.57 \\
"Fire fighters” & 1.64 & 0.71 & 1.41 & 0.47 \\
Supervisors & 0.92 & 0.38 & 0.82 & 0.27 \\
\hline
\end{tabular}

The theoretical range for mean score for each sub-scale ranges from 1 to 4 , with higher score associated with higher attribute for the corresponding grandparenting role dimension. 
TABLE 4. Grandparenting Role Changes and Life Satisfaction

\begin{tabular}{lccl}
\hline & \multicolumn{2}{c}{ Life Satisfaction } & \\
Grandparenting Roles & CG & NCG & F-value \\
& Mean(SD) & Mean(SD) & \\
\hline Daily life helpers (CG=123, NCG=42) & $4.48(2.91)$ & $3.77(2.80)$ & 1.86 \\
Educators (CG=97, NCG=64) & $4.65(2.97)$ & $3.69(2.67)$ & $4.37^{*}$ \\
Advisors (CG=80, NCG=83) & $3.91(3.05)$ & $4.69(2.69)$ & $2.95^{\mathrm{a}}$ \\
“Fire fighters” (CG=66, NCG=99) & $4.87(2.90)$ & $3.91(2.83)$ & $4.44^{*}$ \\
Supervisors (CG=48, NCG=116) & $4.48(2.77)$ & $4.19(2.94)$ & 0.38 \\
\hline a $p<.10 ; * p<.05$. & & &
\end{tabular}


TABLE 5. Hierarchical Regression of Life Satisfaction

\begin{tabular}{|c|c|c|c|c|c|c|c|c|c|}
\hline & \multicolumn{3}{|c|}{ Model 1 (n=159) } & \multicolumn{3}{|c|}{ Model 2 (n=154) } & \multicolumn{3}{|c|}{ Model 3 (n=150) } \\
\hline & $\mathrm{B}$ & SE & Beta & $\mathrm{B}$ & SE & Beta & $\mathrm{B}$ & SE & Beta \\
\hline Education (0/1) & -0.3 & .44 & -.01 & -.45 & .40 & -.08 & -.42 & .39 & -.07 \\
\hline Marital status (0/1) & .14 & .46 & .02 & -.26 & .41 & -.04 & -.36 & .42 & -.06 \\
\hline Living alone & -1.77 & 0.68 & $-0.20 * * *$ & -1.10 & 0.62 & $-0.13^{\mathrm{a}}$ & -0.87 & 0.61 & -0.10 \\
\hline Living in residential home & -2.66 & 0.49 & $-0.46 * * *$ & -1.38 & 0.51 & $-0.24 * * *$ & -1.07 & 0.52 & $-0.19 *$ \\
\hline LSNS & & & & 0.04 & 0.03 & 0.15 & 0.04 & 0.03 & 0.14 \\
\hline Tradition & & & & 0.31 & 0.09 & $0.25 * * *$ & 0.30 & 0.09 & $0.25 * * *$ \\
\hline Self-rated health & & & & 0.48 & 0.22 & $0.16^{*}$ & 0.56 & 0.22 & $0.18^{*}$ \\
\hline Self-rated financial adequacy & & & & 0.55 & 0.23 & $0.17^{*}$ & 0.43 & 0.23 & $0.13^{\mathrm{a}}$ \\
\hline \multicolumn{10}{|l|}{ Grandparenting role changes } \\
\hline Daily life helpers & & & & & & & 0.67 & 0.49 & 0.10 \\
\hline Educators & & & & & & & 0.61 & 0.47 & 0.10 \\
\hline Advisors & & & & & & & -1.46 & 0.45 & $-0.25 * *$ \\
\hline "Fire fighters" & & & & & & & 0.70 & 0.48 & 0.12 \\
\hline Supervisors & \multirow{2}{*}{\multicolumn{3}{|c|}{$\begin{array}{l}\text { Adjusted } \mathrm{R}^{2}=.16(p \\
<.0001)\end{array}$}} & \multirow{2}{*}{\multicolumn{3}{|c|}{$\begin{array}{l}\text { Adjusted } \mathrm{R}^{2}=.34, \mathrm{R}^{2} \\
\text { change }=.20(p<.0001)\end{array}$}} & -0.18 & 0.47 & -0.03 \\
\hline & & & & & & & \multicolumn{3}{|c|}{$\begin{array}{l}\text { Adjusted } \mathrm{R}^{2}=.38, \mathrm{R}^{2} \\
\text { change }=.05(p<.05)\end{array}$} \\
\hline
\end{tabular}

Education ( $0=$ no formal education; 1 = formal education); Marital status ( $0=$ currently

married; 1 = currently not married); Grandparent role changes ( $0=$ no changes; $1=$

decreased as compare to ten years ago); ${ }^{a} p<.10 ;{ }^{*} p<.05 ; * * p<.01 ; * * * p<.001$. 